\title{
Extensions to Reactive Self-Collision Avoidance for Torque and Position Controlled Humanoids
}

\author{
Alexander Dietrich*, Thomas Wimböck ${ }^{*}$, Holger Täubig ${ }^{\dagger}$, Alin Albu-Schäffer ${ }^{*}$, and Gerd Hirzinger \\ *Institute of Robotics and Mechatronics, German Aerospace Center (DLR), Oberpfaffenhofen \\ ${ }^{\dagger}$ German Research Center for Artificial Intelligence, Bremen \\ Contact: Alexander.Dietrich@dlr.de
}

\begin{abstract}
One of the fundamental demands on robotic systems is a safe interaction with their environment. For fulfilling that condition, both collisions with obstacles and the own structure have to be avoided. We address the problem of selfcollisions and propose an algorithm for its avoidance which is based on artificial repulsion potential fields and applicable to both torque and position controlled manipulators. To this end, we design a damping that incorporates the configuration dependance of the robot. For a maximum level of safety, an additional emergency brake strategy based on kinetic energy considerations is introduced for situations in which selfcollisions are not avoidable by the controller. Experiments are performed on DLR's humanoid Justin.
\end{abstract}

\section{INTRODUCTION}

For robots in human and industrial environment, it is crucial to ensure a high level of safety. That involves the avoidance of collisions with both obstacles and the own structure. Over the last decades, this safety aspect has been addressed frequently. A wide variety of approaches has been developed to cope with dynamic environments and unexpected events in the workspace of the manipulator. They range from collision detection methods [1], [2], [3] to reactive path-planning of safe trajectories and repulsion from potential obstacles [4], [5], [6].

An elegant real-time approach has been applied to the HRP-2 humanoid robot [7]. Based on cost functions, collisions are avoided via control in the velocity domain. In [8], an algorithm is presented which predicts a human's motion in the working area of the robot based on estimated velocities. Then, an optimal trajectory for the manipulator is planned to prevent the predicted collision. A wide field of research focuses on reactive repulsion potential field-based designs, introduced by Khatib [9]. This concept has been implemented on the humanoid ASIMO [10]. Therein, virtual repulsive forces are generated between potentially colliding links and transformed via an admittance into corresponding joint motions which are projected into the nullspace of the main task. Another application for potential fields in collision avoidance can be found in [11]. On the Ranger Dexterous Manipulator, repulsive potentials with respect to obstacles, joint limits and singularities in the configuration space are designed. One can say that most of these related approaches have in common that repulsive forces between links are transformed into appropriate joint motions in order to be applicable to the respective position controlled system. However,
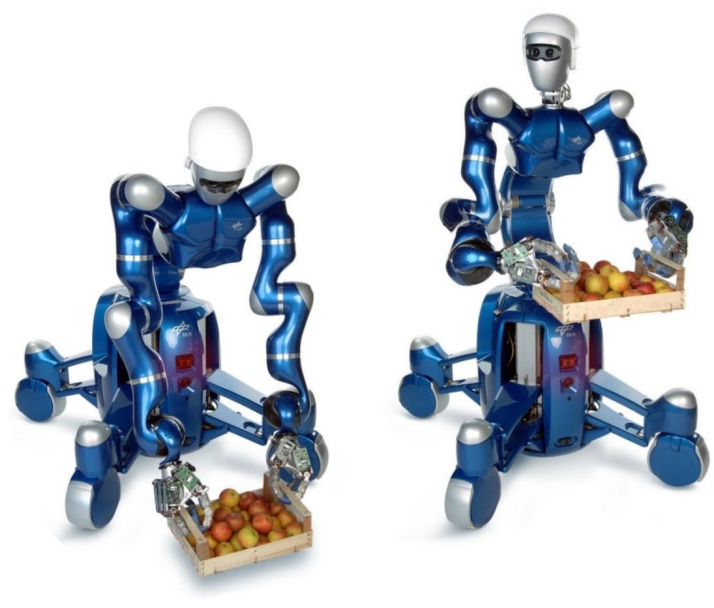

Fig. 1. DLR's humanoid Justin performing a grasping/lifting task.

also for torque controlled manipulators like the upper body of DLR's humanoid Justin [12] collision avoidance applications have been developed. The potential field-based algorithm in [13] leads to joint torques for avoiding self-collisions though leaving the open question of an appropriate damping design and cases of emergency in which the potential fields are overstrained and not able to completely avoid a collision in the end.

The aim of this paper is to present a new repulsion potential field-based algorithm for self-collision avoidance which extends the work started in [13]. The method is implemented on DLR's humanoid Justin, see Fig. 1. One main contribution of the paper is an efficient damping design which incorporates the configuration dependance of collision-endangered situations. Thereby, even critical situations like entangled arms in front of the chest, in which many virtual contact points exist, do not lead to oscillations. The second major contribution is a strategy for indispensable emergency stops of the entire system. Based on the kinetic energy which is stored within the motion of two approaching links, we determine whether a collision is preventable by the repulsion potential fields or mechanical braking is the only possibility left. Thus, the manipulator's safe working range can be enlarged and a maximum level of self-protection is reached. The computation of the reflected, one-dimensional inertia in contact direction is essential for both damping design and 
TABLE I

SYSTEM OVERVIEW

\begin{tabular}{|c||c|c|}
\hline Subsystem & DoF & control mode \\
\hline \hline Torso & 3 & torque \\
\hline Arms & $2 \times 7$ & torque \\
\hline Hands & $2 \times 12$ & torque \\
\hline Head \& Neck & 2 & position \\
\hline Platform \& Legs & 8 & position \\
\hline
\end{tabular}

emergency behavior. The third contribution is an admittancebased interface to position controlled subsystems which can be embedded naturally in the derived force/torque-based design approach. Further, we use a small-sized generic collision model volume representation for distance computation which allows a tighter modeling of the robot than in [13].

The paper is organized as follows. After a brief introduction of the employed system in Section II, we deliver insight into the applied distance computation algorithm which provides the controller with information about potentially colliding links in real-time in Section III. Subsequently, we derive the control algorithm in Section IV. Starting with the design of the potential fields and repulsive forces, we proceed to formulate the damping term. Thereafter, a passage addresses a subset of position controlled joints by introducing an admittance interface. Eventually, a kinetic energy-based logic for emergency cases is proposed. To validate the theoretical claims, experimental results on DLR's humanoid Justin are presented and discussed.

\section{SYSTEM OVERVIEW}

DLR's humanoid Justin consists of an upper body system which is mounted on a mobile platform, see Fig. 1. The latter is able to realize any translational and rotational trajectory. Furthermore, the footprint is variable due to four extendable legs. The upper body consists of a torso, two arms, two hands and a sensor head which is mounted on a pan-tiltunit for unrestricted stereo vision in the working range of the manipulator. The 51 degrees of freedom (DoF) of Justin are grouped by subsystem and control mode in Table I.

The upper body weighs approximately $45 \mathrm{~kg}$, whereas the mobile platform amounts to about $150 \mathrm{~kg}$. Further details on the structure, kinematics and control can be found in [12] (upper body) and [14], [15] (mobile platform).

\section{DISTANCE COMPUTATION}

This chapter describes the solution to a prerequisite task for the repulsion potential field-based algorithm, namely finding the $n_{p}$ pairs of Justin's links having the smallest distances. As collisions are most likely to appear between the links having small distances, these are the objects of interest. The task is to provide the distance and the potential contact points, i.e., the points on these links having that minimal distance, for each of these $n_{p}$ pairs. The algorithm combines a compact and numerically well-posed volume representation with a standard distance computation technique for convex hulls [2].

\section{A. Overview}

Each of Justin's rigid links is modeled by a fixed volume $V$. The computation operates as follows: In every control cycle, all volumes are transformed into the world frame by applying the corresponding transformations. Then, for every pair of links, the distance and corresponding proximal points are determined. It is possible to exclude some pairs from being processed, e.g. because they can not collide. Finally, the $n_{p}$ pairs of smallest distance are kept for further processing.

\section{B. Representation}

The collision model volume of each link has to be a superset of the real volume of that link. Though, it should be as tight as possible and its representation should use as few points as possible for minimizing the computation time for world frame transformations and distance calculations which are dependent on the number of points in the representation.

To do so, we represent volumes in terms of spherically extended convex hulls (swept sphere volumes):

$$
\begin{aligned}
& V\left(r ;\left\{\boldsymbol{p}_{k}\right\}_{k=1}^{n}\right)= \\
& =\left\{\boldsymbol{p}_{r}+\sum_{k=1}^{n} \lambda_{k} \boldsymbol{p}_{k} \mid \lambda_{k} \geq 0 \forall k, \sum_{k=1}^{n} \lambda_{k}=1,\left\|\boldsymbol{p}_{r}\right\| \leq r\right\} \\
& \text { with } r>0, \quad \boldsymbol{p}_{k} \in \mathbb{R}^{3} \forall k .
\end{aligned}
$$

Thus, each volume is the Minkowski-sum of a convex polyhedron, given by the convex hull of $\left\{\boldsymbol{p}_{k}\right\}_{k=1}^{n}$, and a sphere of radius $r$.

This representation is very flexible. It allows modeling of edged and round links using just a few points. Overall, the 28 links of Justin's collision model contain only 78 points and 28 radii. A single link's representation uses only 2.8 points on average, which is very little, e.g., a tetrahedron, which is the smallest polyhedron having a volume different from zero, takes 4 points, and a cuboid takes 8 . Nevertheless, the collision model is tight, see Fig. 2. This is a substantial extension to the presentation used in [13].

\section{Distance and Potential Contact Point Computation}

In each of Justin's joint frames $C_{j}$ the collision volume $V_{j}=V\left(r^{j} ;\left\{\boldsymbol{p}_{k}^{j}\right\}_{k=1}^{n_{j}}\right)$ of the link connected to joint $j$ is defined. The transformations $\boldsymbol{T}_{w \leftarrow j}(\boldsymbol{q})$ of every joint frame $C_{j}$ into world frame $w$ w.r.t. the joint DoF $\boldsymbol{q}$ derive from the robot's kinematics. Using these transformations, the collision model volumes $V_{j}$ are transformed into the world frame by transforming their generating points.

$$
V_{j}^{w}=V\left(r^{j} ;\left\{\boldsymbol{T}_{w \leftarrow j}(\boldsymbol{q}) \boldsymbol{p}_{k}^{j}\right\}_{k=1}^{n_{j}}\right) .
$$

Now, for every pair of volumes $V_{i}^{w}$ and $V_{j}^{w}$, their distance $d_{i, j}$ and corresponding proximal points, also denoted as the contact point pair, are computed.

Distance and potential contact points of the volumes given by the convex hulls of $\left\{\boldsymbol{p}_{k}^{i}\right\}_{k=1}^{n_{i}}$ and $\left\{\boldsymbol{p}_{k}^{j}\right\}_{k=1}^{n_{j}}$ are determined using the algorithm by Gilbert, Johnson, and Keerthi [2].

\footnotetext{
${ }^{1}\left\{\boldsymbol{p}_{k}\right\}_{k=1}^{n}$ describes the quantity of $n$ points $\left\{\boldsymbol{p}_{1}, \ldots, \boldsymbol{p}_{n}\right\}$ defining the
} 

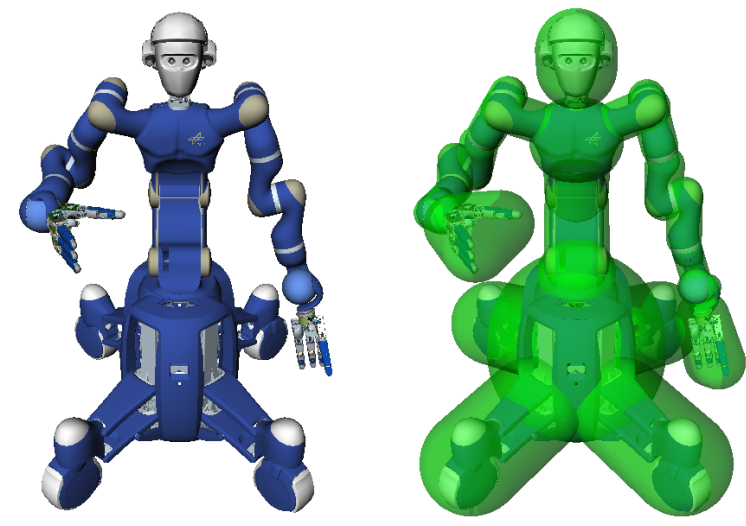

Fig. 2. Self-collision avoidance model of DLR's humanoid Justin consisting of 28 convex hulls (left arm: 8, right arm: 8, mobile platform: 5, torso: 4, head: 2, floor: 1).

This algorithm is widely used and has shown to be efficient and reliable. It yields a distance $d_{i, j}^{\prime}$ and corresponding points $\boldsymbol{x}_{i}^{\prime w}$ and $\boldsymbol{x}_{j}^{\prime w}$. The value $d_{i, j}^{\prime}$ is the minimum distance of any two points taken from the convex hulls conv $\left\{\boldsymbol{p}_{k}^{i}\right\}_{k=1}^{n_{i}}$ and $\operatorname{conv}\left\{\boldsymbol{p}_{k}^{j}\right\}_{k=1}^{n_{j}}$. The points $\boldsymbol{x}_{i}^{\prime w}$ and $\boldsymbol{x}_{j}^{\prime w}$ are placed on these convex hulls such that $d_{i, j}^{\prime}=\left\|\boldsymbol{x}_{j}^{\prime w}-\boldsymbol{x}_{i}^{\prime w}\right\|$.

Subsequently, distance and potential contact points of $V_{i}^{w}$ and $V_{j}^{w}$ are calculated. As $V\left(r ;\left\{\boldsymbol{p}_{k}\right\}_{k=1}^{n}\right)$ enlarges conv $\left\{\boldsymbol{p}_{k}\right\}_{k=1}^{n}$ by $r$ in any direction, the distance between the volumes shrinks by $r^{i}$ due to the enlargement of $V\left(r^{i} ;\left\{\boldsymbol{p}_{k}^{i}\right\}_{k=1}^{n_{i}}\right)$ and by $r^{j}$ due the enlargement of $V\left(r^{j} ;\left\{\boldsymbol{p}_{k}^{j}\right\}_{k=1}^{n_{j}}\right)$. Hence,

$$
d_{i, j}=d_{i, j}^{\prime}-r^{i}-r^{j} .
$$

Due to the enlargements, the potential contact points move on the connecting line of $\boldsymbol{x}_{i}^{\prime w}$ and $\boldsymbol{x}_{j}^{\prime w}$ by $r^{i}$ and $r^{j}$ towards each other.

$$
\begin{aligned}
& \boldsymbol{x}_{i}^{w}=\boldsymbol{x}_{i}^{\prime w}+r^{i} \boldsymbol{v} \text { with } \boldsymbol{v}=\frac{\boldsymbol{x}_{j}^{\prime w}-\boldsymbol{x}_{i}^{\prime w}}{\left\|\boldsymbol{x}_{j}^{\prime w}-\boldsymbol{x}_{i}^{\prime w}\right\|}, \\
& \boldsymbol{x}_{j}^{w}=\boldsymbol{x}_{j}^{\prime w}-r^{j} \boldsymbol{v} .
\end{aligned}
$$

In case of any $d_{i, j}<0$, a collision is present and should be handled appropriately.

While computing distances and contact points, the $n_{p}$ pairs having smallest distance are stored. So far, all contact points are expressed in world coordinates but they are required to be in the respective link's joint frame for self-collision avoidance. Actually, we need both contact points of a link pair in both links' joint frames. So finally, we compute them for the $n_{p}$ pairs

$$
\begin{aligned}
\boldsymbol{x}_{i}^{i} & =\boldsymbol{T}_{w \leftarrow i}^{-1}(\boldsymbol{q}) \cdot \boldsymbol{x}_{i}^{w}, \\
\boldsymbol{x}_{j}^{i} & =\boldsymbol{T}_{w \leftarrow i}^{-1}(\boldsymbol{q}) \cdot \boldsymbol{x}_{j}^{w}, \\
\boldsymbol{x}_{i}^{j} & =\boldsymbol{T}_{w \leftarrow j}^{-1}(\boldsymbol{q}) \cdot \boldsymbol{x}_{i}^{w}, \\
\boldsymbol{x}_{j}^{j} & =\boldsymbol{T}_{w \leftarrow j}^{-1}(\boldsymbol{q}) \cdot \boldsymbol{x}_{j}^{w} .
\end{aligned}
$$

For the real-time self-collision avoidance application, Justin's collision model is calculated once per control cycle
(1 ms) applying 302 pairs of links. With a computing time of 0.3 to $0.4 \mathrm{~ms}$ on an Intel Core2Duo Processor T7400 $(2.16 \mathrm{GHz})$, the distance computation algorithm reveals to be very fast. It is faster than grid-based distance computation approaches like [16] and comparable to those using boundingvolume hierarchies such as [17] and [18]. But it avoids the usage of bounding-volume hierarchies which could induce discontinuities in distance and contact points for link pairs over time. That would complicate the design of a reactive behavior.

\section{DESIGN OF THE CONTROL ALGORITHM}

\section{A. General Methodology}

The basic idea of the control algorithm is to apply repulsion potential fields to approaching links in order to avoid self-collisions. Another main aspect is to take energy out of the system by dissipating kinetic energy via an efficient damping design. In general, the torques which have to be commanded to the robot joints can be expressed as

$$
\boldsymbol{\tau}_{c m d}=-\left(\frac{\partial E_{\mathrm{rep}, \mathrm{tot}}(\boldsymbol{q})}{\partial \boldsymbol{q}}\right)^{T}-\boldsymbol{D}(\boldsymbol{q}) \dot{\boldsymbol{q}}+\boldsymbol{g}(\boldsymbol{q}),
$$

wherein $E_{\text {rep,tot }}$ represents the total potential energy of all $n_{p}$ potential fields applied to the manipulator:

$$
E_{\text {rep }, \text { tot }}(\boldsymbol{q})=\sum_{i=1}^{n_{p}} E_{\text {rep }, i, j}(\boldsymbol{q}) .
$$

Damping in (10) is injected via $\boldsymbol{D}(\boldsymbol{q}) \dot{\boldsymbol{q}}$ and gravity compensation via $\boldsymbol{g}(\boldsymbol{q})$.

The repulsive effect can be described as a function of the distance $d_{i, j}$ between two proximate points $\boldsymbol{x}_{i}$ and $\boldsymbol{x}_{j}$ on different links, see Fig. 3.

$$
\begin{aligned}
\frac{\partial E_{\mathrm{rep}, i, j}(\boldsymbol{q})}{\partial \boldsymbol{q}} & =\frac{\partial E_{\mathrm{rep}, i, j}}{\partial d_{i, j}} \cdot \frac{\partial d_{i, j}}{\partial \boldsymbol{x}_{i, j}} \cdot \frac{\partial \boldsymbol{x}_{i, j}}{\partial \boldsymbol{q}} \\
& =\frac{\partial E_{\mathrm{rep}, i, j}}{\partial d_{i, j}}\left[\frac{\partial d_{i, j}}{\partial \boldsymbol{x}_{i}} \frac{\partial d_{i, j}}{\partial \boldsymbol{x}_{j}}\right]\left[\begin{array}{ll}
\frac{\partial \boldsymbol{x}_{i}}{\partial \boldsymbol{q}_{i}} & \frac{\partial \boldsymbol{x}_{i}}{\partial \boldsymbol{q}_{j}} \\
\frac{\partial \boldsymbol{x}_{j}}{\partial \boldsymbol{q}_{i}} & \frac{\partial \boldsymbol{x}_{j}}{\partial \boldsymbol{q}_{j}}
\end{array}\right]
\end{aligned}
$$

Herein, vectors $\boldsymbol{q}_{i}$ and $\boldsymbol{q}_{j}$ denote the joint values which directly $^{2}$ affect the location of $\boldsymbol{x}_{i}$ and $\boldsymbol{x}_{j}$, respectively. Notice that in general, the contact points may have the same base of the kinematic chain so that $\boldsymbol{q}_{i}$ and $\boldsymbol{q}_{j}$ would overlap. However, just the joints after the branch-off point are relevant. It follows from the multiplication of $\partial d_{i, j} / \partial \boldsymbol{x}_{i, j}$ and $\partial \boldsymbol{x}_{i, j} / \partial \boldsymbol{q}$ that just the principal diagonal of $\partial \boldsymbol{x}_{i, j} / \partial \boldsymbol{q}$ affects the result. The residual multiplications result in zero since the factors are always orthogonal. As an example, let us consider $\partial d_{i, j} / \partial \boldsymbol{x}_{j}$ and $\partial \boldsymbol{x}_{j} / \partial \boldsymbol{q}_{i}$ in the context of Fig. 3. The direction of $\partial d_{i, j} / \partial \boldsymbol{x}_{j}$ is orthogonal to the surface of the link on which $\boldsymbol{x}_{j}$ is lying, whereas $\boldsymbol{q}_{i}$ is only able to let $\boldsymbol{x}_{j}$ move on this surface (indirect influence).

Hence, one needs just to account for direct influence, whereas indirect coupling vanishes.

\footnotetext{
${ }^{2}$ Indirect influence implies the point's motion due to the motion of the corresponding contact point partner.
} 


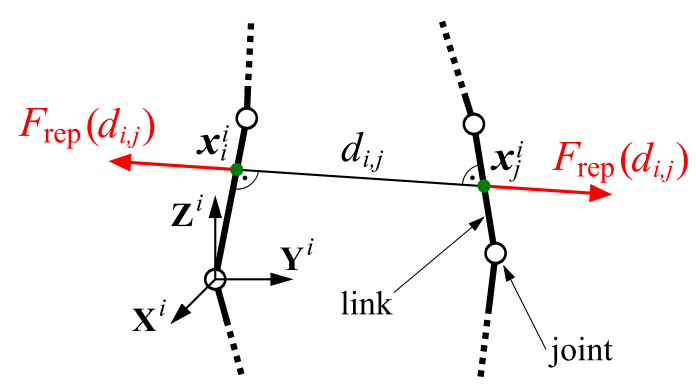

Fig. 3. Relations between corresponding contact points $\boldsymbol{x}_{i}^{i}$ and $\boldsymbol{x}_{j}^{i}$ and resulting repulsive forces $F_{\text {rep }}\left(d_{i, j}\right)$. (subscript: point number; superscript: coordinate frame).

\section{B. Repulsion Potential Field and Repulsive Force}

For us, exclusively the distance $d_{i, j}$ between two points is important for determining forces which repel the links they are placed on. Figure 3 depicts a generic case with $\boldsymbol{x}_{i}^{i}$ and $\boldsymbol{x}_{j}^{i}$ representing the contact point pair. The value $F_{\text {rep }}\left(d_{i, j}\right)$ describes the corresponding repulsive force w.r.t. the distance

$$
d_{i, j}=\left\|\boldsymbol{x}_{i}^{i}-\boldsymbol{x}_{j}^{i}\right\|=\left\|\boldsymbol{x}_{j}^{j}-\boldsymbol{x}_{i}^{j}\right\| .
$$

Subscripts denote the point number and superscripts the coordinate frame in which the points are expressed.

The forces result from repulsion potential fields which are located around the convex hulls discussed in the previous section. We propose the following piecewise function which describes a spherical field with respect to $d_{i, j}$.

$$
E_{\text {rep }}\left(d_{i, j}\right)=\left\{\begin{array}{ll}
-\frac{F_{\max }}{3 d_{\text {sart }}^{\text {2art }}}\left(d_{i, j}-d_{\text {start }}\right)^{3} & \forall d_{i, j} \leq d_{\text {start }} \\
0 & \forall d_{i, j}>d_{\text {start }}
\end{array} .\right.
$$

The corresponding repulsive force is derived as follows:

$$
\begin{aligned}
F_{\text {rep }}\left(d_{i, j}\right) & =-\frac{\partial E_{\text {rep }}\left(d_{i, j}\right)}{\partial d_{i, j}} \\
& = \begin{cases}\frac{F_{\max }}{d_{\text {start }}^{2}}\left(d_{i, j}-d_{\text {start }}\right)^{2} & \forall d_{i, j} \leq d_{\text {start }} \\
0 & \forall d_{i, j}>d_{\text {start }}\end{cases}
\end{aligned}
$$

Figure 4 depicts the corresponding curves. For distances smaller than $d_{\text {start }}$, a growing repulsive force results up to the maximum force $F_{\max }$, while the local stiffness also increases. Obviously, two parameters, namely $F_{\max }$ and $d_{\text {start }}$, are needed to be set in order to define a unique potential and force, respectively. One choice is to limit the maximum local stiffness $\left.\left(\partial F_{\text {rep }}\left(d_{i, j}\right) / \partial d_{i, j}\right)\right|_{d_{i, j}=0}$ to a yet feasible value which is determined by the sample time of the controller. Since we will have multiple contact point pairs and configuration dependent relations between repulsive force and corresponding joint torques, the choice of $F_{\max }$ is in general not easy. However, a rough estimation for the worst case, i.e., one single contact point pair for a collision endangered area/situation, can be made. Hence, a separate $F_{\max }$ for each link can be determined according to the maximum feasible joint torques.
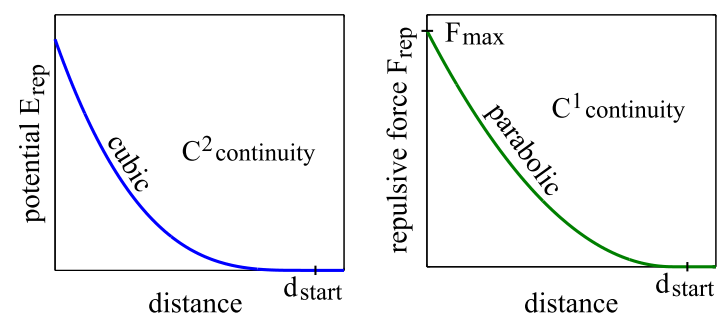

Fig. 4. Potential and repulsive force.

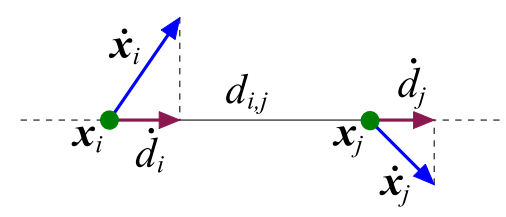

Fig. 5. Illustration of the projected motions of $\boldsymbol{x}_{i}$ and $\boldsymbol{x}_{j}$ into distancedirection. Positive directions are defined from $\boldsymbol{x}_{i}$ to $\boldsymbol{x}_{j}$.

\section{Damping Design}

The aim of this section is to derive a damping design that can be specified by a damping ratio $\zeta$, and that is a function of the system's inertia matrix and the stiffness.

We address the contact point pair $\left(\boldsymbol{x}_{i}^{i}, \boldsymbol{x}_{j}^{i}\right)$ again as depicted in Fig. 3. In addition to the repulsive force, a separate damping force shall be applied to each contact point with respect to the relative motion between the two corresponding contact points. That results in $2 n_{p}$ damping forces or $\boldsymbol{D}(\boldsymbol{q}) \dot{\boldsymbol{q}}$ from (10), respectively.

We define $\dot{d}_{i}$ and $\dot{d}_{j}$ which denote the velocities of the respective contact points projected into distance-direction as depicted in Fig. 5. Positive directions for both $\dot{d}_{i}$ and $\dot{d}_{j}$ are defined from $\boldsymbol{x}_{i}$ to $\boldsymbol{x}_{j}$.

The following closed loop equation shall be satisfied:

$$
\begin{aligned}
& \boldsymbol{M}_{d, i, j}(\boldsymbol{q})\left[\begin{array}{l}
\ddot{d}_{i} \\
\ddot{d}_{j}
\end{array}\right]+\boldsymbol{D}_{d, i, j}\left[\begin{array}{c}
\dot{d}_{i} \\
\dot{d}_{j}
\end{array}\right]+ \\
& \quad+F_{\text {rep }}\left(d_{i, j}\right)\left[\begin{array}{r}
1 \\
-1
\end{array}\right]+\boldsymbol{C}(\boldsymbol{q}, \dot{\boldsymbol{q}})=0 .
\end{aligned}
$$

Coriolis and centrifugal effects are represented by $\boldsymbol{C}(\boldsymbol{q}, \dot{\boldsymbol{q}})$. The mass matrix $\boldsymbol{M}_{d, i, j}$ contains the projections of the reflected inertias at the contact points into distance-direction:

$$
\boldsymbol{M}_{d, i, j}(\boldsymbol{q})=\left[\begin{array}{cc}
M_{d, i}(\boldsymbol{q}) & 0 \\
0 & M_{d, j}(\boldsymbol{q})
\end{array}\right] .
$$

The damping matrix $\boldsymbol{D}_{d, i, j} \in \mathbb{R}^{2 \times 2}$ in (16) is supposed to be chosen such that a desired damping behavior, i.e., damping ratios $\zeta_{1}$ and $\zeta_{2}$, can be realized. The following derivation transforms the known mass matrix of the manipulator into $M_{d, i}(\boldsymbol{q})$. The scalar $M_{d, j}(\boldsymbol{q})$ is attained analogously.

1) Mass Projection $M_{d, i}(\boldsymbol{q})$ : We consider the general relation between joint torques $\tau$ and angular accelerations $\ddot{q}$ of the joints:

$$
\boldsymbol{M}(\boldsymbol{q}) \ddot{\boldsymbol{q}}=\boldsymbol{\tau}
$$

wherein $\boldsymbol{M}(\boldsymbol{q})$ is the corresponding mass matrix. Additionally, the well-known transformation from joint space to 
Cartesian space via the Jacobian matrix $\boldsymbol{J}_{x, i}(\boldsymbol{q})$ with respect to an arbitrary point $\boldsymbol{x}_{i}^{i}(\boldsymbol{q}) \in \mathbb{R}^{3 \times 1}$ is required:

$$
\begin{aligned}
\dot{\boldsymbol{x}}_{i}^{i} & =\boldsymbol{J}_{x, i}(\boldsymbol{q}) \dot{\boldsymbol{q}}, \\
\boldsymbol{\tau} & =\boldsymbol{J}_{x, i}(\boldsymbol{q})^{T} \boldsymbol{F}_{x, i}^{i} .
\end{aligned}
$$

The value $\dot{\boldsymbol{x}}_{i}^{i}$ denotes the Cartesian velocity and $\boldsymbol{F}_{x, i}^{i}$ an external force applied at $\boldsymbol{x}_{i}^{i}$. As our goal is to refer to the distance-direction, we define the coordinates

$$
\boldsymbol{e}_{i}=\frac{\boldsymbol{x}_{j}^{i}-\boldsymbol{x}_{i}^{i}}{d_{i, j}}
$$

with the corresponding Jacobian

$$
\boldsymbol{J}_{d, i}\left(\boldsymbol{e}_{i}\right)=\boldsymbol{e}_{i}^{T},
$$

wherein $\boldsymbol{e}_{i}$ describes the normalized direction vector from $\boldsymbol{x}_{i}^{i}$ to $\boldsymbol{x}_{j}^{i}$. Considering (19), one can project $\dot{\boldsymbol{x}}_{i}^{i}$ into the distancedirection via $\boldsymbol{J}_{d, i}\left(\boldsymbol{e}_{i}\right)$ :

$$
\dot{d}_{i}=\boldsymbol{J}_{d, i}\left(\boldsymbol{e}_{i}\right) \dot{\boldsymbol{x}}_{i}^{i}=\underbrace{\boldsymbol{J}_{d, i}\left(\boldsymbol{e}_{i}\right) \boldsymbol{J}_{x, i}(\boldsymbol{q})}_{\boldsymbol{J}_{i}} \dot{\boldsymbol{q}}
$$

with $\boldsymbol{J}_{i}$ expressing the resultant Jacobian matrix which relates the joint space to the distance-space. Deriving $\dot{d}_{j}$ is done analogously with $\boldsymbol{e}_{j}$ being oriented in the same direction as $\boldsymbol{e}_{i}$.

Based on (23), the acceleration constraint is obtained by differentiation w.r.t. time:

$$
\ddot{d}_{i}=\boldsymbol{J}_{i}(\boldsymbol{q}) \ddot{\boldsymbol{q}}+\dot{\boldsymbol{J}}_{i}(\boldsymbol{q}) \dot{\boldsymbol{q}} .
$$

Combining (18), (20), (23) and (24) leads to

$$
\ddot{d}_{i}=\underbrace{\boldsymbol{J}_{i}(\boldsymbol{q}) \boldsymbol{M}(\boldsymbol{q})^{-1} \boldsymbol{J}_{i}(\boldsymbol{q})^{T}}_{M_{d, i}(\boldsymbol{q})^{-1}} F_{x, i}^{d}+\dot{\boldsymbol{J}}_{i}(\boldsymbol{q}) \dot{\boldsymbol{q}} .
$$

Herein $M_{d, i}(\boldsymbol{q})$ expresses the scalar mass of point $\boldsymbol{x}_{i}^{i}$ to be accelerated in distance-direction. Due to the transformation, $F_{x, i}^{d}$ now describes a scalar force acting at $\boldsymbol{x}_{i}^{i}$.

2) Damping Matrix $\boldsymbol{D}_{d, i, j}$ : Now having $\boldsymbol{M}_{d, i, j}(\boldsymbol{q})$, we can apply desired damping ratios $\zeta_{1}, \zeta_{2}$ to (16) via $\boldsymbol{D}_{d, i, j}$. However, since (16) is nonlinear, we linearize around the working point $d_{i, j}^{*}=f\left(\boldsymbol{q}^{*}\right), \dot{d}_{i}^{*}=\dot{d}_{j}^{*}=0$, under the additional assumption of a quasi-static analysis. This leads to

$$
\boldsymbol{M}_{d, i, j}\left(\boldsymbol{q}^{*}\right)\left[\begin{array}{l}
\delta \ddot{d}_{i} \\
\delta \ddot{d}_{j}
\end{array}\right]+\boldsymbol{D}_{d, i, j}^{*}\left[\begin{array}{l}
\delta \dot{d}_{i} \\
\delta \dot{d}_{j}
\end{array}\right]+\boldsymbol{K}_{d, i, j}^{*}\left[\begin{array}{l}
\delta d_{i} \\
\delta d_{j}
\end{array}\right]=0,
$$

wherein $\boldsymbol{K}_{d, i, j}^{*}$ denotes the local stiffness matrix:

$$
\begin{aligned}
\boldsymbol{K}_{d, i, j}^{*}\left(d_{i, j}^{*}\right) & =\left.\left[\begin{array}{r}
1 \\
-1
\end{array}\right] \frac{\partial F_{\mathrm{rep}}\left(d_{i, j}\right)}{\partial d_{i, j}}\right|_{d_{i, j}=d_{i, j}^{*}} \cdot \frac{\partial d_{i, j}}{\partial\left[d_{i} d_{j}\right]} \\
& =\left.\frac{\partial^{2} E_{\mathrm{rep}}\left(d_{i, j}\right)}{\partial d_{i, j}^{2}}\right|_{d_{i, j}=d_{i, j}^{*}} \cdot\left[\begin{array}{rr}
1 & -1 \\
-1 & 1
\end{array}\right] .
\end{aligned}
$$

The local damping behavior is represented by $\boldsymbol{D}_{d, i, j}^{*}$. Based on (26) and (27), various methods from linear algebra theory can be applied in order to realize the desired damping ratios. We have chosen the Double Diagonalization approach, see

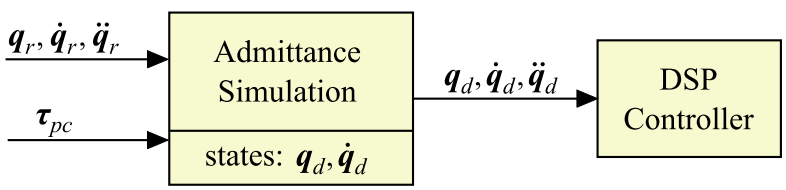

Fig. 6. Admittance Simulation.

[19] for more details. Finally, the required damping matrix can be formally written as

$$
\boldsymbol{D}_{d, i, j}^{*}\left(\boldsymbol{q}^{*}\right)=\mathcal{D}\left(\boldsymbol{M}_{d, i, j}\left(\boldsymbol{q}^{*}\right), \boldsymbol{K}_{d, i, j}^{*}\left(d_{i, j}^{*}\right), \zeta_{1}, \zeta_{2}\right) .
$$

It will be computed and applied at each time instant.

\section{Collision Avoidance Torques}

The procedure described up to now has to be applied to each of the $n_{p}$ contact point pairs. The collision avoidance torques $\boldsymbol{\tau}_{\text {coll }}$ due to repulsion and damping effects w.r.t. the actual values $\boldsymbol{q}, d_{i, j}, \dot{d}_{i}$ and $\dot{d}_{j}$ equal

$$
\boldsymbol{\tau}_{\text {coll }}=\sum_{i=1}^{n_{p}}\left[\begin{array}{c}
\boldsymbol{J}_{i}(\boldsymbol{q}) \\
\boldsymbol{J}_{j}(\boldsymbol{q})
\end{array}\right]^{T}\left(\left[\begin{array}{c}
-F_{\text {rep }}\left(d_{i, j}\right) \\
F_{\text {rep }}\left(d_{i, j}\right)
\end{array}\right]-\boldsymbol{D}_{d, i, j}^{*}\left[\begin{array}{c}
\dot{d}_{i} \\
\dot{d}_{j}
\end{array}\right]\right)
$$

with $j$ defined such that it is the corresponding contact point number to $i$.

\section{E. Admittance Interface}

The computed torque $\tau_{\text {coll }}$ has to be split up:

$$
\boldsymbol{\tau}_{\text {coll }}=\left(\begin{array}{c}
\boldsymbol{\tau}_{t c} \\
\boldsymbol{\tau}_{p c}
\end{array}\right) .
$$

$\tau_{t c} \in \mathbb{R}^{n_{t c}}$ expresses the torques for all $n_{t c}$ torque controlled joints, whereas $\boldsymbol{\tau}_{p c} \in \mathbb{R}^{n_{p c}}$ contains the torques for the $n_{p c}$ position controlled DoF. For each position controlled joint, the respective torque has to be transformed into a desired joint motion, comprised in $\boldsymbol{q}_{d}$. We suggest an admittance interface as follows:

$$
\boldsymbol{M}_{p c}(\boldsymbol{q})\left(\ddot{\boldsymbol{q}}_{d}-\ddot{\boldsymbol{q}}_{r}\right)+\boldsymbol{D}_{p c}\left(\dot{\boldsymbol{q}}_{d}-\dot{\boldsymbol{q}}_{r}\right)+\boldsymbol{K}_{p c}\left(\boldsymbol{q}_{d}-\boldsymbol{q}_{r}\right)=\boldsymbol{\tau}_{p c} .
$$

Herein $\boldsymbol{M}_{p c}(\boldsymbol{q})$ comprises the $n_{p c}$ inertias with respect to the corresponding joints. $\boldsymbol{D}_{p c}$ and $\boldsymbol{K}_{p c}$ represent arbitrary positive definite matrices for damping and spring behavior of the admittance loop. The vector $\boldsymbol{q}_{r} \in \mathbb{R}^{n_{p c}}$ denotes the reference joint values to accomplish the nominal task, whereas $\boldsymbol{q}_{d} \in \mathbb{R}^{n_{p c}}$ contains the desired commanded values under consideration of the additional collision avoidance behavior. Figure 6 depicts the general methodology. In the case of Justin, each joint is torque controlled except for the mobile platform and the two neck joints which are responsible for pan and tilt motions of the head.

\section{F. Controller Output}

Beside the joint motions from the admittance loop which have to be commanded by the position controllers, all $n_{t c}$ torques have to be applied to the torque controlled robot joints to satisfy (10):

$$
\boldsymbol{\tau}_{c m d}=\boldsymbol{\tau}_{t c}+\boldsymbol{g}(\boldsymbol{q}) .
$$




\section{G. Energy-based Emergency Stop Algorithm}

During normal operation, collisions are improbable if the repulsion fields are active and appropriately parameterized. However, an emergency stop which leads to a mechanical braking of each motor is yet an additional safety feature, though, an emergency stop shall only occur if it is indispensable and should not constrict the usual working range.

One possible approach is to engage the brakes if a minimum emergency distance is reached. In this context, let us consider two different operating states. Firstly, we address the case of a slow, highly precise manipulation task like filling a glas with water. During the whole operation, the manipulator will execute quasi-static motions. On the contrary, a task such as ball catching requires trajectories which account for dynamic effects. Although a high accuracy is still essential, rapid movements of the manipulator are top priority now. Certainly, more kinetic energy will be stored in the system during the task. As a consequence, an abrupt mechanical braking of all actuators would cause overshootings due to compliances located in the structure and the drive system. In the first operating state, the emergency distance may be set comparatively low, primarily accounting for the accuracy of the collision model. In the case of the ball catching task, it is both necessary to determine if the repulsion fields are powerful enough to avoid a collision and to account for overshootings if the brakes get activated otherwise. Apparently, the emergency distance should be dependent on the kinetic energy of the approaching links and the expected overshoots due to braking.

1) Limit Case for Avoidable Self-Collisions via the Repulsion Potential Fields: We address the kinetic energy which is stored in a contact point pair or in the corresponding links, respectively. However, we do not take into account the total inertias and velocities of the two contact points but only the projected masses imposed in (16) and the velocities in distance-direction.

$$
E_{\text {pair }, i, j}=\frac{1}{2}\left(g\left(\dot{d}_{i}\right) M_{d, i}(\boldsymbol{q}) \dot{d}_{i}^{2}+g\left(-\dot{d}_{j}\right) M_{d, j}(\boldsymbol{q}) \dot{d}_{j}^{2}\right)
$$

with

$$
g(z)=\left\{\begin{array}{ll}
1 & \forall z \geq 0 \\
0 & \forall z<0
\end{array},\right.
$$

wherein $g(z)$ is used to determine the direction of motion of the respective contact point and to neglect the energy if the point goes away from the corresponding contact partner. This is due to the prevention of negative energy values.

$E_{\text {pair }, i, j}$ describes the instantaneous kinetic energy which is stored in the approaching motion of the contact points. In other words, $E_{\mathrm{pair}, i, j}$ is the energy which has to be taken out of the system in order to avoid a collision of the considered links.

On the other side, the potential energy

$$
E_{\text {stored }}\left(d_{i, j}\right)=E_{\text {rep }}(0)-E_{\text {rep }}\left(d_{i, j}\right)
$$

is stored within the potential field and leads to a consequential reduction of $E_{\mathrm{pair}, i, j}$. One can say that the repulsion potential is too weak if $E_{\text {stored }}<E_{\text {pair }, i, j}$. Therefore, a variable and permanently adjusted kinetic energy-based distance, denoted $d_{\mathrm{ke}, i, j}$, can be obtained from the limit case:

$$
d_{\mathrm{ke}, i, j}=\left\{\begin{array}{ll}
E_{\text {stored }}^{-1}\left(E_{\mathrm{pair}, i, j}\right) & \forall E_{\mathrm{pair}, i, j} \leq E_{\mathrm{rep}}(0) \\
d_{\text {start }} & \forall E_{\mathrm{pair}, i, j}>E_{\mathrm{rep}}(0)
\end{array} .\right.
$$

The inverse function is feasible since the potential described by $E_{\text {rep }}\left(d_{i, j}\right)$ is strictly monotonically decreasing.

2) Decelerating the Links before Braking: However, just engaging the brakes if $d_{i, j}<d_{\mathrm{ke}, i, j}$ could result in the following undesirable event: Once a potential field is entered while much kinetic energy is stored within the approaching links, the discussed criterion is reached promptly and leads to an abrupt stop although the actual distance $d_{\text {start }}$ is comparatively large. Correspondingly, the kinetic energy has to be absorbed by the brakes and the structure. A more preferable behavior would be an extensive, active deceleration of the links and an emergency stop in the last safe time instant such that overshooting due to elasticities does not lead to a self-collision. In this context, a second distance $d_{\mathrm{os}, i, j}(\boldsymbol{q}, \dot{\boldsymbol{q}})$ can be determined which describes the maximum overshoot of abruptly stopped links w.r.t. the configuration and joint velocities. To attain a suitable value, measurements can be performed [20].

Between $d_{\mathrm{ke}, i, j}$ and the last safe distance $d_{\mathrm{os}, i, j}$, the maximum force $F_{\max }$ is applied, taking out of the system as much kinetic energy as possible.

3) Geometric Minimum Distance: We impose a third distance $d_{\text {geom }}$ which takes into account the accuracy of the distance computation algorithm, the sample time and model uncertainties. This constant specifies the minimum distance between two contact points which may not be gone below in all cases.

4) Emergency Behavior: Finally, an emergency logic can be set up, incorporating the three discussed distances:

Action $= \begin{cases}\text { Engage brakes } & \text { if } d_{i, j} \leq d_{\mathrm{geom}}+d_{\mathrm{os}, i, j} \\ \text { Apply } F_{\max } & \text { if } d_{\mathrm{geom}}+d_{\mathrm{os}, i, j}<d_{i, j} \leq d_{\mathrm{ke}, i, j} . \\ \text { No operation } & \text { otherwise }\end{cases}$

\section{RESULTS}

In the following, experiments for the validation of the proposed control structure will be presented. All along, the manipulator is controlled via an impedance controller [19] in gravity compensation mode ${ }^{3}$. The user is required to feed kinetic energy into the system manually. A maximum of $n_{p}=25$ multiple contact point pairs is applied during the experiment which complies with the design such that each penetrated potential field is taken into account and no discontinuities arise in the commanded torques. Figure 7 (left) depicts the starting position. Starting distance $d_{\text {start }}$ is set to $0.15 \mathrm{~m}$ and maximum repulsive force $F_{\max }=25 \mathrm{~N}$. Damping ratios $\zeta_{1}$ and $\zeta_{2}$ are equal and set to $\zeta$ which is varied in the following.

\footnotetext{
${ }^{3}$ Except for the left arm (7 DoF), all other joints are locked in the present experiment.
} 

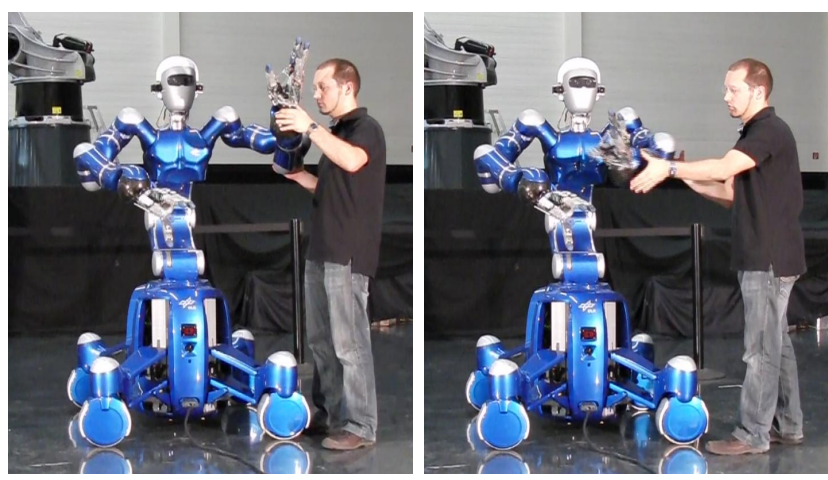

Fig. 7. Starting position (left) and snapshot during the experiment (right).

The user throws the left forearm onto the right arm as shown in Fig. 7 (right) in the case of undamped $(\zeta=0)$, underdamped $(\zeta=0.7)$, critically damped $(\zeta=1.0)$ and overdamped $(\zeta=1.3)$ design. In this experiment several contact pairs generate repulsion. Figure 8 depicts the two most interesting ones which are placed on the left hand and right hand links (left column plots) and on the left hand and right wrist links (right column plots).

As the user brought in about the same amount of kinetic energy in all of the four scenarios ${ }^{4}$, the penetration of the potential fields is significantly smaller while damping is active. Evidently, the returning velocities of the links are affected by $\zeta$ as it can be seen in the upper plots in Fig. 8. Thus, without dissipation by damping forces, no energy is taken out of the system. It is also noticeable, that the highest damping $(\zeta=1.3)$ does not lead to the smallest penetration in the second contact point pair (left hand and right wrist). The reason is that the interaction between the multiple contact point pairs results in different behavior of the left arm.

Due to the potential design, the repulsive forces are continuously differentiable ( $\mathrm{C}^{1}$ continuity, see Fig. 4$)$. Since the damping directly depends on the local stiffness, the damping forces are continous but not continuously differentiable as it can be seen in the bottom plots in Fig. 8 .

Beside the depicted contact point pairs in Fig. 8, several repulsions between potentially colliding links emerge during the experiment, performing analogously with respect to the applied damping ratio. In the considered case, a total number of 14 contact point pairs is relevant for selfcollision avoidance. In the most critical case (undamped), the most crucial contact point pair (left hand and right hand) consumes $25.2 \%$ of the total potential energy which is stored in the corresponding repulsion field until the distance reaches its minimum at time $0.28 \mathrm{~s}$ and increases again. Hence, a sufficient buffer is left and switching to $F_{\max }$ or engaging the brakes, as discussed in (37), is not necessary.

The video which is attached to this paper shows several exemplary scenes from a presentation of the control

\footnotetext{
${ }^{4}$ The energies which are absorbed by the most crucial potential field in all four cases, i.e., between left hand and right hand, have a maximum deviation of $<12 \%$.
}
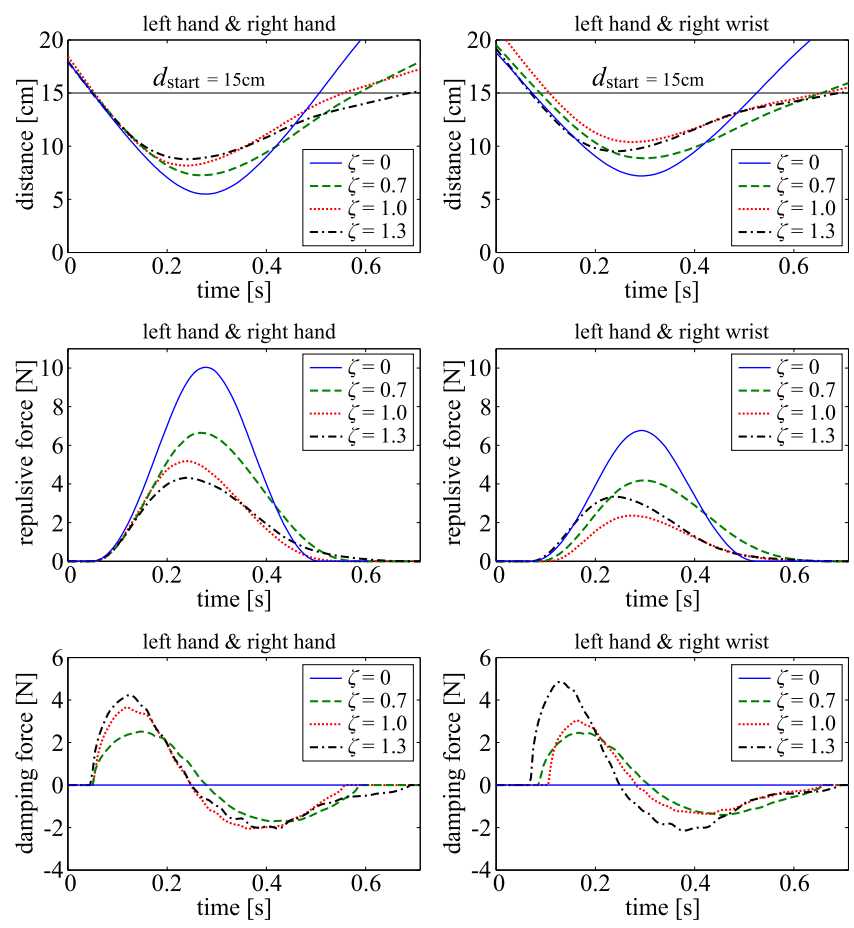

Fig. 8. Repulsion between left hand and right hand/wrist. (no damping $\zeta=0$ : blue, solid; underdamped $\zeta=0.7$ : green, dashed; critically damped $\zeta=1.0$ : red, dotted; overdamped $\zeta=1.3$ : black, chain dotted).

algorithm to the public at the Automatica Fair 2010 in Munich. Moreover, the reactive behavior in the case of different damping ratios (undamped, underdamped, critically damped) is visualized as well as the admittance interface concerning the position controlled head joints. A short scene also demonstrates the manipulator's behavior if the brakes are engaged during motion.

\section{CONCLUSION AND FUTURE WORKS}

In robotics, a high level of safety and reliability is only ensured if self-collision can be completely excluded. In this paper, we discussed and analyzed this issue and proposed a controller to remedy this problem. Based on the wellknown technique of repulsion potential fields placed around the robot links, the segments are repelled from each other to avoid self-collisions. An effective damping design was introduced which incorporates the configuration dependent inertia of the manipulator. After deriving the necessary joint torques, we included an admittance interface for position controlled subsystems of the robot. As the fundamental issue is to guarantee a maximum level of protection, we proposed an emergency stop algorithm that incorporates the kinetic energy which is stored within approaching links and the assumed overshoot due to elasticity while braking. This problem becomes even more relevant for more compliant systems with larger dynamics such as the recently developed DLR hand arm system. The emergency logic is restricted to cases in which self-collisions are unavoidable. Thereby, the working range of the manipulator is not limited unnecessarily. Experimental results on DLR's humanoid manipulator 
Justin supported our approach.

Future work will focus on the enlargement of the algorithm to the point of collision avoidance with obstacles/humans in the working range of the robot. An efficient real-time identification and detection of potential obstacles is essential for that purpose. Further improvements can also be achieved in terms of the potential field design. A configuration dependent potential field design would take advantage of the use of the full feasible torque range and minimize the necessity of emergency stops.

\section{REFERENCES}

[1] J. Kuffner, K. Nishiwaki, S. Kagami, Y. Kuniyoshi, M. Inaba, and H. Inoue, "Self-Collision Detection and Prevention for Humanoid Robots", in Proc. of the 2002 IEEE International Conference on Robotics and Automation, pp. 2265-2270, May 2002.

[2] E. G. Gilbert, D. W. Johnson, and S. S. Keerthi, "A Fast Procedure for Computing the Distance between Complex Objects in 3D Space", IEEE Journal on Robotics and Automation, Vol. 4, No. 2, 1988.

[3] S. Haddadin, A. Albu-Schäffer, A. De Luca, and G. Hirzinger, "Collision Detection \& Reaction: A Contribution to Safe Physical Human-Robot Interaction", in Proc. of the 2008 IEEE-RSJ International Conference on Intelligent Robots and Systems, pp. 3356-3363, September 2008.

[4] F. Seto, K. Kosuge, and Y. Hirata, "Self-collision Avoidance Motion Control for Human Robot Cooperation System using RoBE", in Proc. of the 2005 IEEE-RSJ International Conference on Intelligent Robots and Systems, pp. 50-55, August 2005.

[5] H. Liu, X. Deng, and H. Zha, "A Planning Method for Safe Interaction between Human Arms and Robot Manipulators", in Proc. of the 2005 IEEE-RSJ International Conference on Intelligent Robots and Systems, pp. 1814-1820, August 2005.

[6] E. Freund and J. Rossmann, "The basic Ideas of a proven Dynamic Collision Avoidance Approach for Multi-Robot Manipulator Systems", in Proc. of the 2003 IEEE-RSJ International Conference on Intelligent Robots and Systems, pp. 1173-1177, October 2003.

[7] O. Stasse, A. Escande, N. Mansard, S. Miossec, P. Evrard, and A. Kheddar, "Real-Time (Self)-Collision Avoidance Task on a HRP-2 Humanoid Robot", in Proc. of the 2008 IEEE International Conference on Robotics and Automation, pp. 3200-3205, May 2008.

[8] L. Balan and G. M. Bone, "Real-time 3D Collision Avoidance Method for Safe Human and Robot Coexistence", in Proc. of the 2006 IEEERSJ International Conference on Intelligent Robots and Systems, pp. 276-282, October 2006.

[9] O. Khatib, "Real-Time Obstacle Avoidance for Manipulators and Mobile Robots", The International Journal of Robotics Research, Vol. 5, No. 1, pp. 90-98, Spring 1986.

[10] H. Sugiura, M. Gienger, H. Janssen, and C. Goerick, "Real-Time Self Collision Avoidance for Humanoids by means of Nullspace Criteria and Task Intervals", in Proc. of the IEEE-RAS International Conference on Humanoid Robots, pp. 575-580, December 2006.

[11] N. Scott and C. R. Carignan, "A Line-Based Obstacle Avoidance Technique for Dexterous Manipulator Operations", in Proc. of the 2008 IEEE International Conference on Robotics and Automation, pp. 3353-3358, May 2008.

[12] C. Borst, C. Ott, T. Wimböck, B. Brunner, F. Zacharias, B. Bäuml, U. Hillenbrand, S. Haddadin, A. Albu-Schäffer, and G. Hirzinger, "A humanoid upper body system for two-handed manipulation", in Proc. of the 2007 IEEE International Conference on Robotics and Automation, pp. 2766-2767, April 2007.

[13] A. De Santis, A. Albu-Schäffer, C. Ott, B. Siciliano, and G. Hirzinger, "The skeleton algorithm for self-collision avoidance of a humanoid manipulator", in Proc. of the 2007 IEEE/ASME International Conference on Advanced Intelligent Mechatronics, 2007.

[14] P. R. Giordano, M. Fuchs, A. Albu-Schäffer, and G. Hirzinger, "On the Kinematic Modeling and Control of a Mobile Platform Equipped with Steering Wheels and Movable Legs", in Proc. of the 2009 IEEE International Conference on Robotics and Automation, pp. 4080-4087, May 2009.
[15] A. Dietrich, T. Wimböck, A. Albu-Schäffer, and G. Hirzinger, "Singularity Avoidance for Nonholonomic, Omnidirectional Wheeled Mobile Platforms with Variable Footprint", in Proc. of the 2011 IEEE International Conference on Robotics and Automation, pp. 6136-6142, May 2011.

[16] J. Himmelstein, E. Ferré, and J.-P. Laumond, "Swept Volume approximation of polygon soups", in Proc. of the 2007 IEEE International Conference on Robotics and Automation, pp. 4854-4860, April 2007.

[17] M. Tang, Y. Kim, and D. Manocha, " $\mathrm{C}^{2} \mathrm{~A}$ : Controlled Conservative Advancement for Continuous Collision Detection of Polygonal Models", in Proc. of the 2009 IEEE International Conference on Robotics and Automation, pp. 849-854, May 2009.

[18] S. Redon, Y. Kim, M. Lin, and D. Manocha, "Fast Continuous Collision Detection for Articulated Models", in Proc. of the 9th ACM Symposium on Solid Modeling and Applications, pp. 145-156, June 2004.

[19] A. Albu-Schäffer, C. Ott, and G. Hirzinger, "A Passivity Based Cartesian Impedance Controller for Flexible Joint Robots - Part II: Full State Feedback, Impedance Design and Experiments", in Proc. of the 2004 IEEE International Conference on Robotics and Automation, pp. 2666-2672, April 2004.

[20] S. Haddadin, A. Albu-Schäffer, M. Frommberger, and G. Hirzinger, "The Role of the Robot Mass and Velocity in Physical Human-Robot Interaction - Part II: Constrained Blunt Impacts", in Proc. of the 2008 IEEE International Conference on Robotics and Automation, pp. 13391345, May 2008. 